\title{
Exploring an S-matrix for gravitational collapse II: a momentum space analysis
}

\section{G. Veneziano}

Theory Division, CERN,

CH-1211 Geneva 23, Switzerland, and

Collège de France,

11 place M. Berthelot, 75005 Paris, France

E-mail: gabriele.veneziano@cern.ch

\section{J. Wosiek}

M. Smoluchowski Institute of Physics, Jagellonian University,

Reymonta 4, 30-059 Cracow, Poland

E-mail: wosiek@th.if.uj.edu.pl

ABSTRACT: We complement our earlier position-space exploration of a recently proposed S-matrix for transplanckian scattering by a momentum-space analysis. As in the previous paper, we restrict ourselves to the case of axisymmetric collisions of extended sources. Comparison between the two formulations allows for several cross-checks while showing their complementary advantages. In particular, the momentum-space formulation leads to an easier computation of the emitted-graviton spectra and to an attempt to study the system beyond its critical points into the presumed gravitational-collapse regime.

KeYwords: Black Holes, Models of Quantum Gravity, Spacetime Singularities. 


\section{Contents}

1. Introduction 1

2. Momentum space action and field equations 2

3. Examples of source profiles

4. Numerical solutions and comparison with $x$-space results 5

5. Spectrum of emitted gravitons 7

6. Beyond the critical point: a first attempt 10

\section{Introduction}

In a companion paper [1] we have explored a recent proposal [2] for an $S$-matrix description of transplanckian scattering in four-dimensional spacetime. The proposal is based on an approximate resummation of the semi-classical corrections to the leading eikonal approximation [3] and amounts to solving the equations of motion of an effective action introduced quite sometime ago [4, 5]. In a suitable limit, the longitudinal dynamics can be factored out leaving behind an effective dynamics in the "transverse" two-dimensional space.

In [1] we considered, quite systematically, the case of the axisymmetric (central) collision of two extended sources/beams. This case has several advantaged over the original problem of a two-particle collision at non-vanishing impact parameter:

- The partial differential equations (PDE's) reduce to ordinary differential equations (ODE's) making the problem affordable by analytic and numerical techniques, without having to make either an azimuthal-average approximation [2] or to resort to advanced numerical techniques [6].

- The IR-sensitive graviton polarization, which was neglected by hand in [2], is simply not produced in the axisymmetic case [1].

- We can consider a wide variety of initial states by playing with the many (shape and intensity) parameters chracterizing the sources and check for the existence of critical surfaces in this multidimensional space. The results can then be compared with those coming from closed trapped surface (CTS) criteria [0, 8] and will be tested, hopefully in the near future, against numerical GR calculations (see [9, 10] for a few results already available for this case). 
The results of [1], based on a position-space analysis, gave further support to the conclusions of [2]. In particular, we were able to prove a one-way relation between the CTS criterion of [8] and the criticality condition in the ODE system. We could also determine quite precisely the critical surfaces in a variety of cases and found a good quantitative agreement with CTS-based criteria [8]. Finally, we confirmed that, above those critical lines, a new absorption of the elastic S-matrix turns on with a universal behaviour reminiscent of Choptuik's critical exponent [11].

In this paper we complement our previous work [1] by a momentum-space analysis according to the following outline: in section 2 we present the momentum space formulation of the extended-source problem and, after specializing to the axisymmetric case, we give the explicit form of the action and of the equations of motion. In section 3 we recall some interesting extended sources already considered in [1] adding their momentum-space form. In section 4 we study numerically the field equations, determine the critical lines in parameter space, and compare them with the position-space results of [1]. In section 5 we discuss the spectrum of the emitted gravitons starting from the perturbative regime and until one approaches the critical lines. In section 6 we present an attempt to extend the solutions in the (presumed) BH-phase.

\section{Momentum space action and field equations}

We recall from [1] the position-space action of [2] generalized to extended sources:

$$
\begin{aligned}
\frac{\mathcal{A}}{2 \pi G s}= & \int d^{2} x\left[a(x) \bar{s}(x)+\bar{a}(x) s(x)-\frac{1}{2} \nabla_{i} \bar{a} \nabla_{i} a\right] \\
& -\frac{(\pi R)^{2}}{2} \int d^{2} x\left(\left(\nabla^{2} \phi\right)^{2}+2 \phi\left(\nabla^{2} a \nabla^{2} \bar{a}-\nabla_{i} \nabla_{j} a \nabla_{i} \nabla_{j} \bar{a}\right)\right),
\end{aligned}
$$

with the three real fields $a, \bar{a}$ and $\phi$ representing the two longitudinal and the single, IR-safe, transverse component of the gravitational field, respectively.

The center of mass energy $\sqrt{s}$ provides the overall normalization factor $2 \pi G s=\frac{\pi}{2 G} R^{2}$, while the two sources $s(x), \bar{s}(x)$ are normalized by $\int d^{2} x s(x)=\int d^{2} x \bar{s}(x)=1$. In order to go to momentum space we can either start from (2.1) or generalize directly eq. (5.2) of [2]. The result is:

$$
\begin{aligned}
\frac{\pi A}{G s}= & \int \frac{d^{2} \boldsymbol{k}}{\boldsymbol{k}^{2}}\left[\beta_{1}(\boldsymbol{k}) s_{2}(-\boldsymbol{k})+\beta_{2}(\boldsymbol{k}) s_{1}(-\boldsymbol{k})-\beta_{1}(\boldsymbol{k}) \beta_{2}(-\boldsymbol{k})\right] \\
& -\frac{(\pi R)^{2}}{2} \int d^{2} \boldsymbol{k}\left[\frac{1}{2} h(\boldsymbol{k}) h(-\boldsymbol{k})-h(-\boldsymbol{k}) \mathcal{H}(\boldsymbol{k})\right]
\end{aligned}
$$

where the FT of the sources (still denoted by $s_{i}$ ) are normalized by requiring $s_{i}(0)=1$. Furthermore,

$$
\begin{aligned}
& \beta_{1}(\boldsymbol{k})=\frac{k^{2} a(\boldsymbol{k})}{2}, \quad \beta_{2}(\boldsymbol{k})=\frac{k^{2} \bar{a}(\boldsymbol{k})}{2}, \quad h(\boldsymbol{k})=-k^{2} \phi(\boldsymbol{k}), \\
& \mathcal{H}(\boldsymbol{k}) \equiv \frac{1}{\pi^{2} \boldsymbol{k}^{2}} \int d^{2} \boldsymbol{k}_{1} d^{2} \boldsymbol{k}_{2} \delta\left(\boldsymbol{k}-\boldsymbol{k}_{1}-\boldsymbol{k}_{2}\right) \beta_{1}\left(\boldsymbol{k}_{1}\right) \beta_{2}\left(\boldsymbol{k}_{2}\right) \sin ^{2} \theta_{12}
\end{aligned}
$$


and $\theta_{12}$ is the angle between the two transverse momenta $\boldsymbol{k}_{1}$ and $\boldsymbol{k}_{2}$.

If we now specialize to the axisymmetric case where sources and fields depend only upon $k_{i}^{2}$, we can use the following relations:

$$
\begin{gathered}
\int d^{2} k=\pi \int d k^{2}, \\
\int d^{2} \boldsymbol{k}_{1} d^{2} \boldsymbol{k}_{2} \delta\left(\boldsymbol{k}-\boldsymbol{k}_{1}-\boldsymbol{k}_{2}\right) \frac{\sin ^{2} \theta_{12}}{k^{2}}=\frac{1}{4} \int_{\lambda \geq 0} \frac{d k_{1}^{2} d k_{2}^{2}}{k_{1}^{2} k_{2}^{2} k^{2}} \sqrt{\lambda\left(k_{1}^{2}, k_{2}^{2}, k^{2}\right)},
\end{gathered}
$$

where

$$
\lambda\left(k_{1}^{2}, k_{2}^{2}, k^{2}\right)=\left(2 k_{1}^{2} k_{2}^{2}+2 k^{2} k_{2}^{2}+2 k_{1}^{2} k^{2}-k^{4}-k_{1}^{4}-k_{2}^{4}\right),
$$

and we have used the identity:

$$
\sin ^{2} \theta_{12}=\frac{\lambda\left(k_{1}^{2}, k_{2}^{2}, k^{2}\right)}{4 k_{1}^{2} k_{2}^{2}}
$$

together with the change of variable (at fixed $k^{2}, k_{1}^{2}$ )

$$
d k_{2}^{2}=d\left(k-k_{1}\right)^{2}=2|k|\left|k_{1}\right| \sin \theta_{k 1} d \theta_{k 1}=2|k|_{1}\left|k_{2}\right| \sin \theta_{12} d \theta_{k 1} .
$$

One thus arrives at the final form of the axially-symmetric effective action:

$$
\begin{aligned}
\frac{A}{G s}= & \int \frac{d k^{2}}{k^{2}}\left[\beta_{1}\left(k^{2}\right) s_{2}\left(k^{2}\right)+\beta_{2}\left(k^{2}\right) s_{1}\left(k^{2}\right)-\beta_{1}\left(k^{2}\right) \beta_{2}\left(k^{2}\right)\right] \\
& \left.-\frac{(\pi R)^{2}}{2} \int d k^{2} \frac{1}{2} h\left(k^{2}\right) h\left(k^{2}\right)+\frac{R^{2}}{8} \int \frac{d k^{2} d k_{1}^{2} d k_{2}^{2}}{k_{1}^{2} k_{2}^{2} k^{2}} \sqrt{\lambda\left(k_{1}^{2}, k_{2}^{2}, k^{2}\right)} h\left(k^{2}\right) \beta_{1}\left(k_{1}^{2}\right) \beta_{2}\left(k_{2}^{2}\right) .9\right)
\end{aligned}
$$

whose equations of motion read:

$$
\begin{aligned}
& h\left(k^{2}\right)=\frac{1}{4 \pi^{2}} \int \frac{d k_{1}^{2} d k_{2}^{2}}{k^{2} k_{1}^{2} k_{2}^{2}} \sqrt{\lambda\left(k_{1}^{2}, k_{2}^{2}, k^{2}\right)} \beta_{1}\left(k_{1}^{2}\right) \beta_{2}\left(k_{2}^{2}\right), \\
& \beta_{i}\left(k^{2}\right)=s_{i}\left(k^{2}\right)+\frac{R^{2}}{8} \int \frac{d k_{1}^{2} d k_{2}^{2}}{k_{1}^{2} k_{2}^{2}} \sqrt{\lambda\left(k_{1}^{2}, k_{2}^{2}, k^{2}\right)} h\left(k_{1}^{2}\right) \beta_{i}\left(k_{2}^{2}\right) .
\end{aligned}
$$

It is not a completely trivial exercise to show directly the equivalence of these equations with the corresponding ones in position-space [1] (where a dot stands for $d / d r^{2}$ ),

$$
\begin{aligned}
\dot{a}_{i} & =-\frac{1}{2 \pi \rho(r)} \frac{R_{i}(r)}{R}, \\
\ddot{\rho} & =\frac{1}{2}(2 \pi R)^{2} \dot{a}_{1} \dot{a}_{2}=\frac{1}{2} \frac{R_{1}(r) R_{2}(r)}{\rho^{2}(r)}, \\
R_{i}(r) & =R \int_{|\mathbf{x}|^{2} \leq r^{2}} d^{2} x s_{i}(x) .
\end{aligned}
$$

The proof, not reported here, makes use of the following (known?) integral of three Bessel functions (that we have checked numerically):

$$
\int_{0}^{\infty} d r J_{1}(r k) J_{1}\left(r k_{1}\right) J_{1}\left(r k_{2}\right)=\frac{1}{2 \pi} \frac{\sqrt{\lambda\left(k_{1}^{2}, k_{2}^{2}, k^{2}\right)}}{k k_{1} k_{2}} \Theta(\lambda) .
$$




\section{Examples of source profiles}

In this section we list various extended sources already introduced in [1] and give their momentum representations.

A. As a first class, consider finite-size sources with the following profiles:

$$
s_{1}(\mathbf{x})=s_{2}(\mathbf{x})=\frac{L^{4} d}{\pi\left(L^{4} d+r^{4}(1-d)\right)^{3 / 2}} \Theta(L-r) .
$$

Later, without lack of generality, we shall be fixing the transverse size of the two identical beams $L$ to be 1 . One can easily verify that these sources satisfy our normalizations and that

$$
\pi \int_{0}^{r^{2}} d \rho^{2} s(\rho)=R(r / L) / R=\frac{r^{2}}{\left(L^{4} d+r^{4}(1-d)\right)^{1 / 2}}, \pi \int_{0}^{L^{2}} s(r) d r^{2}=1 .
$$

Once Fourier transformed to momentum space and normalized according to the prescription of section 2 , the above sources become $(k=|\mathbf{k}|)$ :

$$
s_{1}(k)=s_{2}(k)=\frac{\int_{0}^{L^{2}} d r^{2} J_{0}(k r) s(r)}{\int_{0}^{L^{2}} d r^{2} s(r)}=\int_{0}^{L^{2}} d r^{2} J_{0}(k r) \frac{L^{4} d}{\left(L^{4} d+r^{4}(1-d)\right)^{3 / 2}} .
$$

In particular, for two homogeneous beams $(d=1)$ we have:

$$
s_{1}(k)=s_{2}(k)=\int_{0}^{L^{2}} d r^{2} J_{0}(k r)=\frac{2}{k L} J_{1}(k L) .
$$

B. Point-like sources are difficult to deal with numerically, especially in momentum space. We introduce therefore Gaussian-smeared versions of the point and ring-like sources considered in [四]:

$$
\begin{aligned}
s_{1}(\boldsymbol{x}) & =\frac{1}{\mathcal{N}_{1}} \exp \left(-\frac{r^{2}}{2 \sigma^{2}}\right) \Theta\left(L_{1}-r\right), s_{2}(\boldsymbol{x})=\frac{1}{\mathcal{N}_{2}} \exp \left(-\frac{\left(r-L_{2}\right)^{2}}{2 \sigma^{2}}\right) \Theta\left(L_{2}-r\right), \\
\mathcal{N}_{1} & =2 \pi \sigma^{2}\left(1-\exp \left(\frac{-L_{1}^{2}}{2 \sigma^{2}}\right)\right), \mathcal{N}_{2}=2 \pi\left(\sigma^{2}\left(\exp \left(\frac{-L_{2}^{2}}{2 \sigma^{2}}\right)-1\right)+\sigma L_{2} \sqrt{\frac{\pi}{2}} \operatorname{Erf} \frac{L_{2}}{\sqrt{2} \sigma}\right)
\end{aligned}
$$

When $\sigma \rightarrow 0(\infty)$ such configuration reduces to the one of the point-ring (two homogeneous beams) case. The corresponding Fourier transforms are:

$$
s_{i}(\boldsymbol{k})=2 \pi \int_{0}^{L_{i}} r d r J_{0}(k r) s_{i}(\boldsymbol{x}) .
$$

C. Another interesting example is that of gaussian sources concentrated at $r=0$. They correspond to:

$$
s_{i}(\mathbf{x})=\frac{1}{2 \pi L_{i}^{2}} \exp \left(-\frac{r^{2}}{2 L_{i}^{2}}\right), \frac{R_{i}(r)}{R}=1-\exp \left(-\frac{r^{2}}{2 L_{i}^{2}}\right),
$$

or, in momentum space, to:

$$
s_{i}(\mathbf{k})=\exp \left(-\frac{k^{2} L_{i}^{2}}{2}\right) .
$$




\section{Numerical solutions and comparison with $x$-space results}

There are two ways to solve the non-linear system (2.10). One may use an iterative(recursive) procedure suggested by the form of the equations, or treat them (after discretization) as an algebraic system of polynomial equations of third order. The two approaches are to some extent complementary. The recursion turns out to be convergent only in the dispersive phase, and can therefore be used to determine the inter-phase boundary in parameter space. The algebraic approach allows to explore also the $\mathrm{BH}$ phase by generating genuine complex solutions of the system. It can also be cross checked, of course, with the recursive method in the dispersive phase.

Both approaches rely on a momentum discretization procedure. As a first step, we introduce new variables which span unit intervals and are thus convenient for that purpose:

$$
x=\frac{1}{1+k_{1} L}, \quad y=\frac{1}{1+k_{2} L}, \quad v=\frac{1}{1+k L} .
$$

Here $L$ is the size of the two identical sources (A, B from the previous section) or of one of them $\left(L_{1}\right.$ in the case $\left.\mathrm{C}\right)$. In the following we set $L=1$. In the new variables equations 2.10 read:

$$
\begin{aligned}
h(v) & =\frac{1}{\pi^{2}} \int_{T} \frac{d x d y}{x(1-x) y(1-y)} \frac{v^{2}}{(1-v)^{2}} \sqrt{\lambda(x, y, v)} \beta_{1}(x) \beta_{2}(y), \\
\beta_{i}(v) & =s_{i}(v)+\frac{R^{2}}{2} \int_{T} \frac{d x d y}{x(1-x) y(1-y)} \sqrt{\lambda(x, y, v)} h(x) \beta_{i}(y) .
\end{aligned}
$$

At fixed $v$ the $x, y$ integrals are over the triangular region $T$ which is bounded by three hyperbolas

$$
\begin{aligned}
& 0<v<1, \quad 0<x<1, \quad y_{\min }(x, v)<y<y_{\max }(x, v), \\
& y_{\text {min }}(x, v)=\frac{x v}{x+v-x v} \quad y_{\max }(x, v)=\left\{\begin{array}{l}
\frac{x v}{v-x+x v} x<v \\
\frac{x v}{x-v+x v} x \geq v
\end{array}\right.
\end{aligned}
$$

Next, we discretize the variables

$$
u \longrightarrow u_{i}=\frac{1}{2 n}+\frac{i-1}{n}, \quad i=1, \ldots, n, u=x, y, v,
$$

and turn the integral equations into a set of $3 n$ algebraic equations

$$
\begin{aligned}
f_{i} & =s_{i}+\frac{R^{2}}{2} \Sigma_{j, k=1}^{n} w_{i, j, k}^{(b)} f_{2 n+i} f_{i}, \\
f_{n+i} & =s_{n+i}+\frac{R^{2}}{2} \sum_{j, k=1}^{n} w_{i, j, k}^{(b)} f_{2 n+i} f_{n+i}, \\
f_{2 n+i} & =\frac{1}{\pi^{2}} \Sigma_{j, k=1}^{n} w_{i, j, k}^{(h)} f_{i} f_{n+i},
\end{aligned}
$$

where the weights $w$ are the discretized versions of the kernels * measure in the corresponding continuous equations

$$
\begin{aligned}
w^{(b)}(i, j, k) & =\frac{\lambda\left(x_{j}, y_{k}, v_{i}\right)}{x_{j}\left(1-x_{j}\right) y_{k}\left(1-y_{k}\right)} \operatorname{Area}(i, j, k), \\
w^{(h)}(i, j, k) & =\frac{\lambda\left(x_{j}, y_{k}, v_{i}\right)}{x_{j}\left(1-x_{j}\right) y_{k}\left(1-y_{k}\right)\left(1 / v_{i}-1\right)^{2}} \operatorname{Area}(i, j, k),
\end{aligned}
$$




\begin{tabular}{|c|c|c|c|c|c|c|c|}
\hline$d$ & & 0.5 & 1.0 & 1.6 & 2.5 & 4.0 & \\
\hline A-x & & 0.419 & 0.471 & 0.502 & 0.528 & 0.550 & \\
\hline A-p & & 0.429 & 0.476 & 0.499 & 0.501 & 0.477 & \\
\hline$\sigma$ & & 0.01 & 0.1 & 0.2 & 0.3 & 3.0 & \\
\hline B-x & & 0.615 & 0.572 & 0.525 & 0.486 & 0.470 & \\
\hline B-p & & 0.058 & 0.436 & 0.501 & 0.489 & 0.476 & \\
\hline$\rho$ & 0.25 & 0.333 & 0.5 & 1.0 & 2.0 & 3.0 & 4.0 \\
\hline C-X & .810 & .816 & .821 & .823 & .821 & .816 & .810 \\
\hline C-p & .823 & .833 & .850 & 841 & .838 & .840 & .832 \\
\hline
\end{tabular}

Table 1: $(R / L)_{c}$ for a range of sizes of the power-like and Gaussian sources: a comparison between configuration and momentum-space results. A, B and $\mathrm{C}$ label sources as discussed in section 3. In the case $\mathrm{C}: \rho=L_{2} / L_{1}$ and the critical value of the ratio $2 R /\left(L_{1}+L_{2}\right)$ is shown.

and the $\operatorname{Area}(i, j, k)$ is the area of the intersection of a small, $1 / n^{2}$, square with the "triangle" $T$ if the center of the square $\left(x_{j}, y_{k}\right)$ is inside $T$, and zero if the center is outside $T$.

As already remarked, these equations can be used either to set up a recursive procedure, or can be directly solved numerically. The latter approach, dubbed the "algebraic method", works in both the $\mathrm{BH}$ and the dispersive phase.

In the first two (A and $\mathrm{B}$ ) rows of table 1 we compare a sample of critical values of $R$

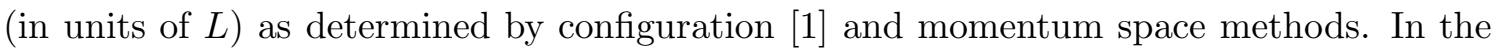
latter $R_{c}$ is defined as a point where the recursion (4.6) diverges. As such it depends on the length of a trajectory $n$ and in principle requires extrapolation to $n=\infty$. In the table we used $n \leq 80$.

For extended sources both approaches are consistent meaning that at $n \sim 50-80$ momentum space estimates have already converged. However, for narrower sources, momentum method requires a yet finer discretization. This is to be expected, since a finite mesh in momentum, say $\Delta p$, limits the spatial resolution to $\Delta x>1 / \Delta p$. In such cases one has to extrapolate numerical data from the case of extended sources as done in [6]. In the following, we shall be discussing only homogenous beams where a finite $n \sim 50$ is adequate. Notice again the two special cases mentioned earlier, namely $d=1(\infty)$ and $\sigma=\infty(0)$ which correspond to the scattering of homogeneous beams and that of a particle and a ring. The critical radii for these cases $\left(R_{c} \sim .47\right.$ and $R_{c}=2^{1 / 2} 3^{-3 / 4} \sim .62$ respectively) were obtained in [2] and agree with the ones quoted in the table except for the momentum study of the infinitely narrow sources which was to be expected.

The third row of the table summarizes the head-on collision of the two central, gaussian sources with different widths $(\mathrm{C})$. The problem is symmetric with respect to the interchange $L_{1} \leftrightarrow L_{2}$ therefore we display $R_{c}$ in units of $\left(L_{1}+L_{2}\right) / 2$. Agreement between x- and p-space methods is quite satisfactory. The configuration space technique used in [1] was manifestly symmetric under the interchange of $L_{1}$ and $L_{2}$ as reflected in the table. However, in the momentum space calculations we have deliberately used only one source size as a scale. The resulting small asymmetry gives an idea of the sensitivity to the discretization parameter 


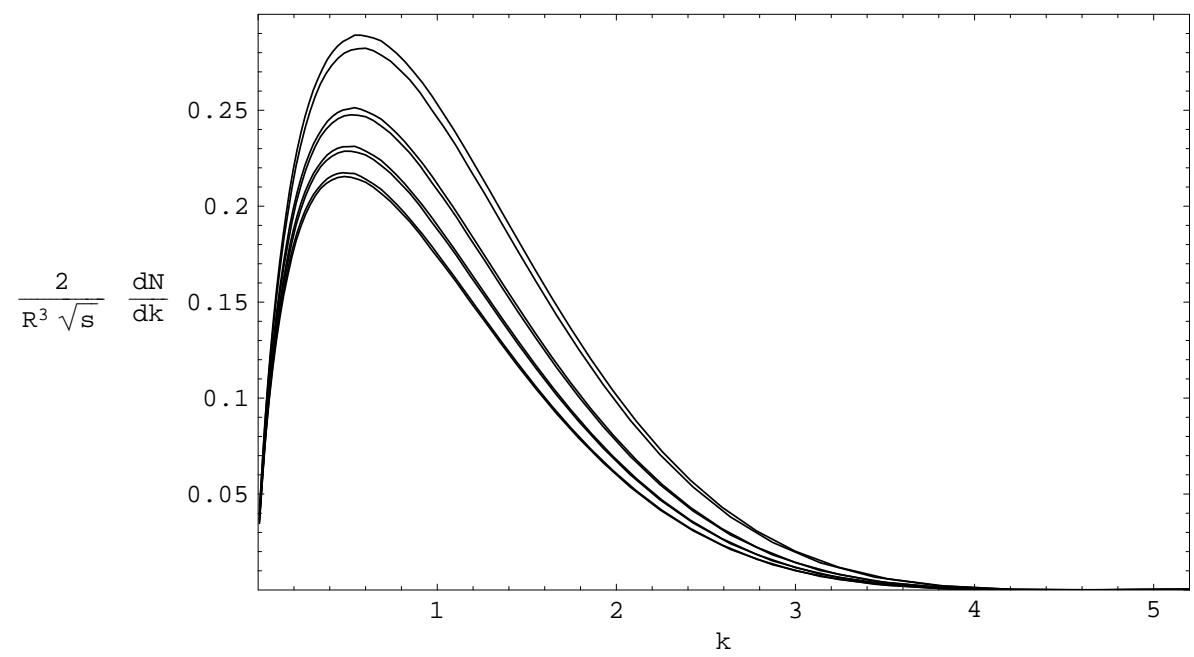

Figure 1: Scattering of two homogeneous beams of size $L_{i}=1$. Inclusive spectra $|\boldsymbol{k} \| h(\boldsymbol{k})|^{2}$ of emitted gravitons, as a function of $|\boldsymbol{k}|$ close to criticality. Bottom to top: $R=0.44,0.45,0.46,0.47$, with $R_{c}=0.470673$. The two curves for each $R$ are for $n=60$ and $n=70$.

$n$ (which was not so large here, $n \leq 20$ ).

Finally, we emphasize a very weak dependence of $\left[2 R /\left(L_{1}+L_{2}\right)\right]_{c}$ on $\rho$. This confirms the observation, made already in [1], that the critical line is remarkably linear in the $\left(L_{1}, L_{2}\right)$ plane indicating that a simple sum $L_{1}+L_{2}$ controls the concentration of energy in a large part of parameter space.

\section{Spectrum of emitted gravitons}

Let us recall, from [2], that the graviton spectrum is determined in terms of $h(k)$ through:

$$
\frac{1}{\sigma_{T}} \frac{d \sigma}{d \boldsymbol{k}^{2} d y} \sim G s R^{2}|h(\boldsymbol{k})|^{2}
$$

In figure 1 we show a suitably normalized transverse-energy distribution of gravitons, $|\boldsymbol{k}| h(\boldsymbol{k})^{2} \sim \frac{d \sigma}{d \mid \boldsymbol{k}}$, as generated from the iterations (4.6). The two adjacent curves for each $R$ give an idea about the residual dependence on the "volume" $n$. As usual, the convergence with $n$ is slower in the vicinity of the critical point, but the figure suggests that the behaviour near $R_{c}$ is rather regular. In particular there is no indication for a buildup of any divergence in the spectrum as $R \rightarrow R_{c}^{-}$.

A similar conclusion follows from figure 2 where the density profile of the gravitational field $(\pi R)^{4} r h(r)^{2}=r(1-\dot{\rho}(r))^{2}$ in transverse distance $r$ is shown. The numerical calculations were done in configuration space following [1]. There is no "finite volume", $n$, in this case and we can concentrate on the dependence on $R$. Again, no singularity in $r$ develops as $R \rightarrow R_{c}$ and we can define a smooth limiting distribution at the critical point $R_{c}^{-}$. Note however, that the dependence on $R$ close to $R_{c}$ is rather strong (the change of $R$ is tiny for the three uppermost curves), suggesting that the limiting distribution is attained with a large, possibly infinite, derivative. 


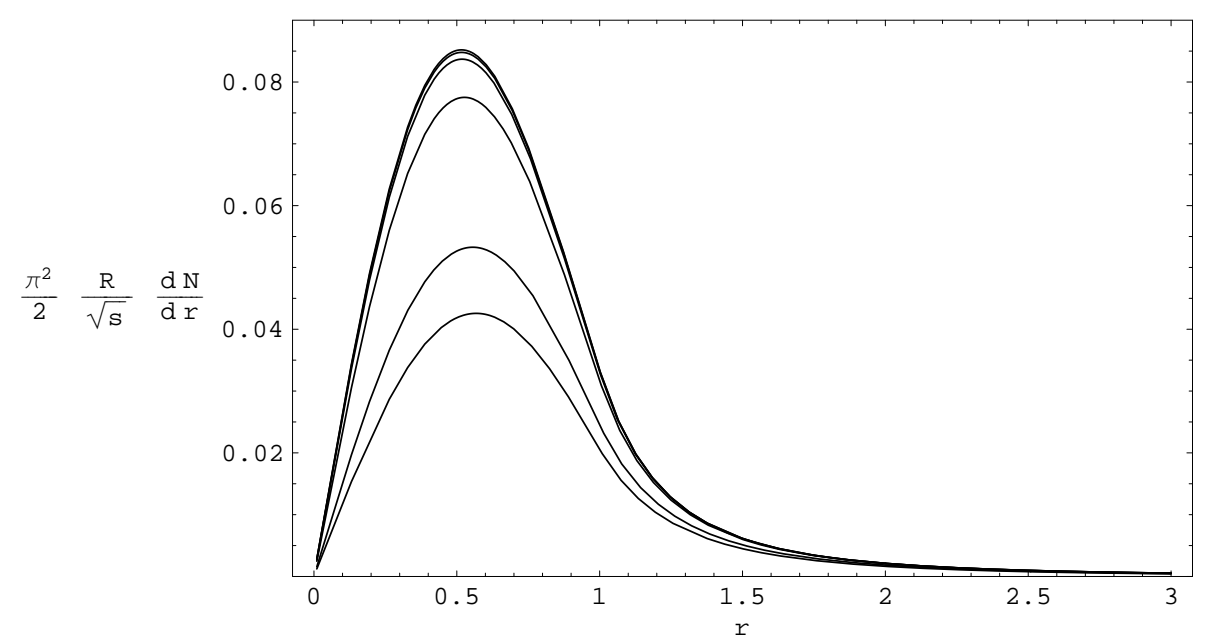

Figure 2: Same as in the previous figure but in configuration space. The various curves (brom bottom up) correspond to $R=0.45,0.46,0.47,0.4706,0.47064,0.47065$, and $R_{c}=0.470673$.

Figure 3 illustrates yet better this point. It turns out that the maximum value of the above density depends on $\left(R_{c}-R\right)$ as a simple square root. When left unconstrained, the best fitted power was always within $\pm 1 \%$ of $1 / 2$, remaining very stable against adding or removing initial/final data points. The solid line in figure 3 shows the fit where the power was actually fixed to $1 / 2$. Similarly, we have found that the width of the distribution also behaves as $c_{1}+c_{2}\left(R_{c}-R\right)^{1 / 2}$, with finite coefficients $c_{1}, c_{2}$. Therefore, indeed, the limiting distribution exists and is approached with an infinite derivative w.r.t. $R$. At the transition point, gravitons are emitted preferentially from half the distance from the source's edges.

In [1] we have already analyzed the total multiplicity of emitted gravitons since it is related to the derivative of the action with respect to $R$. We found that it approaches a finite constant at $R_{c}^{-}$with a square root branch point at $R_{c}$ (see figure 5 of [1]), with a "best fit" given by $0.138-0.46\left(R_{c}-R\right)^{0.523}$. This is hardly surprising given the above results for the differential distribution.

To close the circle we have also compared the Fourier transform of the $p$-space solution $h(k)$ with $h(r)$ obtained directly from the solution $\rho(r)$ in $x$-space. The two agree locally within $2-3 \%$, the discrepancy being caused again by the finite discretization in momentum space. This is reassuring, not only in confirming again the consistency of the whole procedure, but also because, due to the infrared behaviour, transition between $x$ - and $p$ representations is rather subtle. In particular, we find ( $c f$. again figure 1) that the emission amplitude $h(k)$ (and also $\beta_{i}(k)$ ) diverges at small momenta as

$$
h(k) \sim \frac{1}{\sqrt{k}},
$$

and is exponentially damped at large $k$. Consequently, the action (2.9) is IR divergent and, even if the divergence is physically irrelevant ( $c f$. the infinite Coulomb phase), has to be treated with care numerically. 


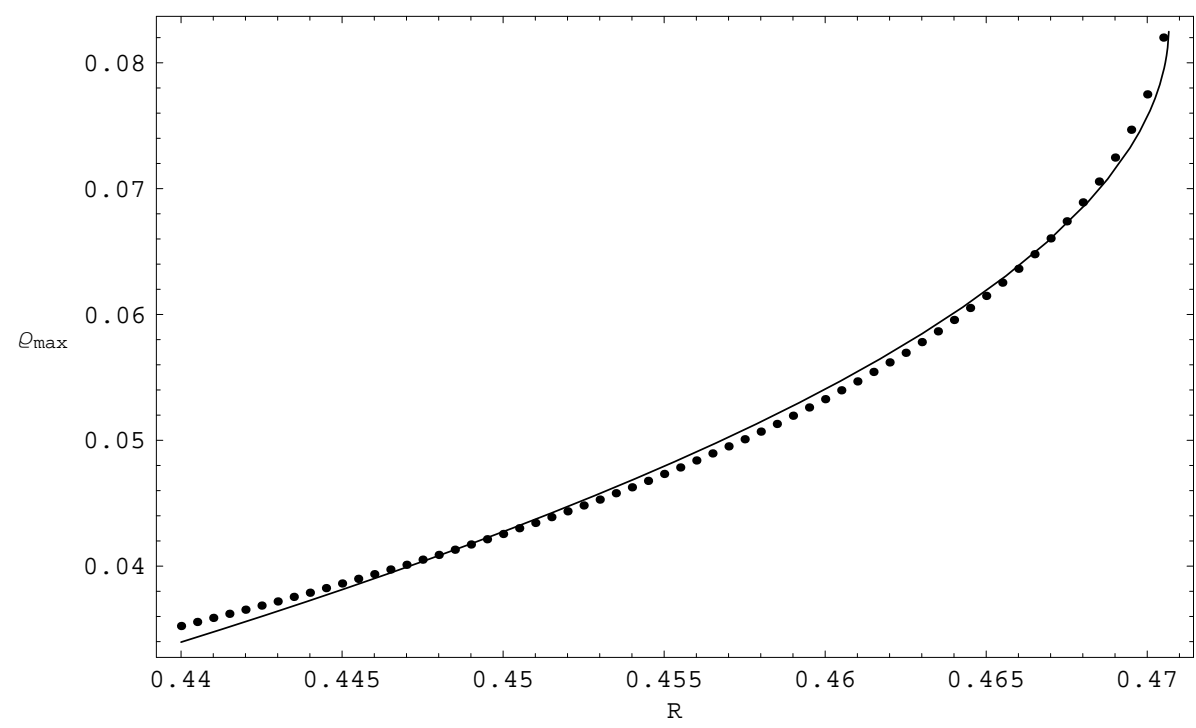

Figure 3: The maximum of the spatial density of gravitons as a function of $R$. Points are from solving eqs. 2.11). The linear fit, $0.083-0.28\left(R_{c}-R\right)^{1 / 2}$, is also shown.

The large $k$ behaviour of the spectrum can be qualitatively assessed from figure 4 . The distribution resembles much more an exponential than the gaussian shape of the sources we have put in (this is the $\mathrm{C}$ case of section 3). Actually, a rather interesting structure emerges, as shown in that figure. Let us use units in which $L=1$ and increase $R$ (i.e. the energy), starting from very small values. In that perturbative region the spectrum appears to consist of two exponentials separated by a "knee", i.e. the slope at small $k$, is smaller than the one at large $k$. However, as we increase $R$ towards its critical value, the knee tends to disappear leaving behind an almost perfect exponential $\exp (-b|k|)$. The slope $b$ of the exponential (determined mainly by $L$ in the perturbative region) now strongly depends on $\left(R-R_{c}\right)$. Figure 5 illustrates the points we just made and indicates that the slope also has a rather singular behaviour, possibly of the form $b \sim c_{1}+c_{2}\left(R-R_{c}\right)^{\gamma}$.

One can finally try to determine the total multiplicity by integrating the spectra (either in $x$ or in $p$ space). At small $R / L$ one finds:

$$
\langle N\rangle \sim G s(R / L)^{2}
$$

Given that the average transverse energy is $O(1 / L)$ this corresponds to an average total transverse energy in the emitted gravitons given by:

$$
\left\langle E_{T}\right\rangle \sim \sqrt{s}(R / L)^{3} \ll \sqrt{s} .
$$

However, as one approaches $R_{c}$, this quantity clearly becomes $O(\sqrt{s})$ possibly implying that the transverse energy becomes a good estimate of the total radiated energy but also, unfortunately, that imposing energy-conservation, i.e. taking into account the back-reaction on the sources, becomes mandatory. 


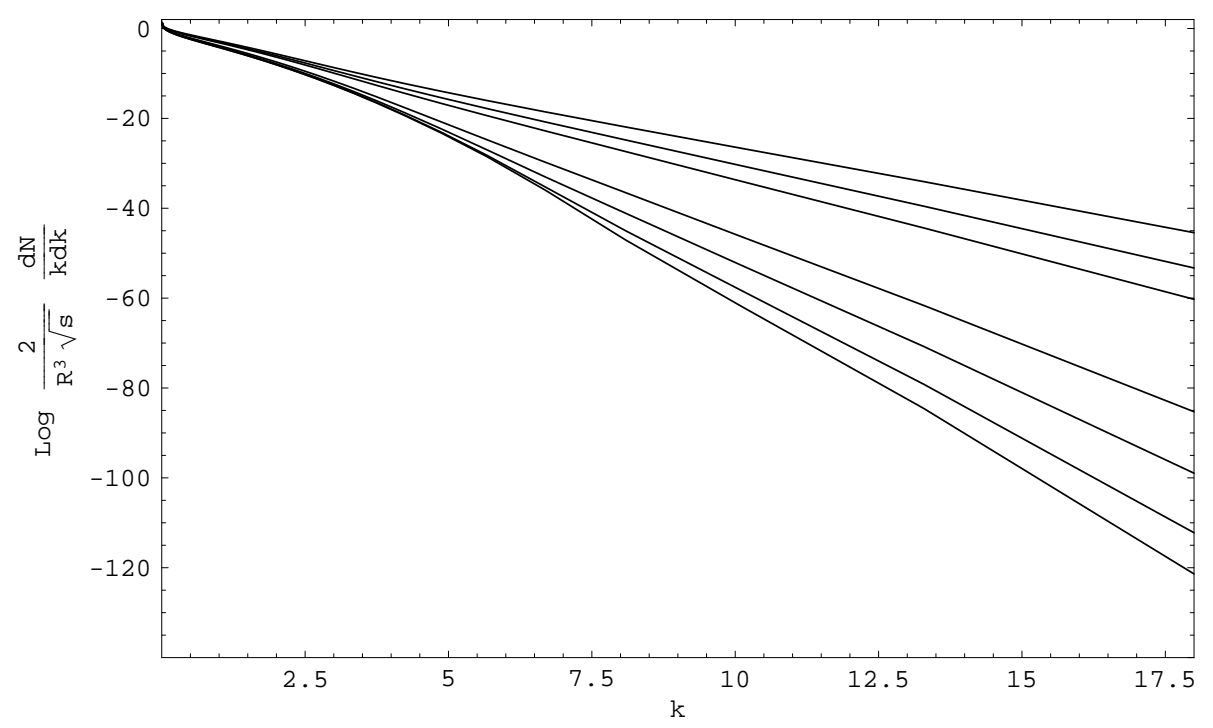

Figure 4: A logarithmic plot of the distribution (5.1) for $R=.10, .20, .40,60, .80, .82, .83$ (bottom to top).

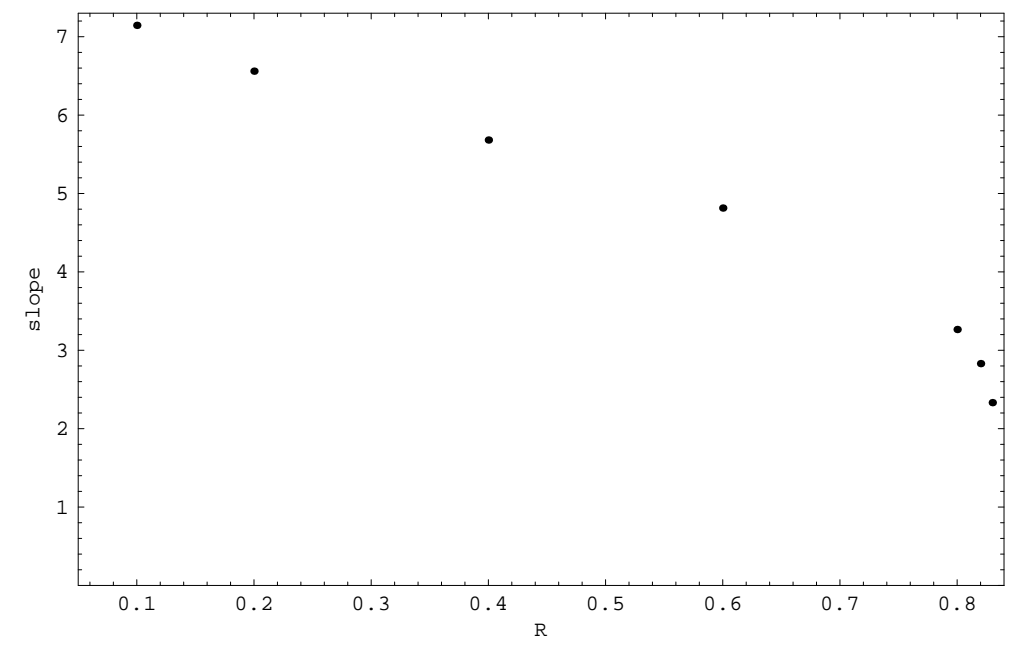

Figure 5: R dependence of the slopes from the previous figure (determined locally from $10<k<$ 12.5).

\section{Beyond the critical point: a first attempt}

There is obviously much interest in extending the numerical analysis into a $\mathrm{BH}$ phase. Although precise solutions for $R>R_{c}$ are not available at this stage, we can get an approximate picture of their complicated structure using the momentum-space approach. As already explained in section 4, the discretized equations of motion (4.2) can be solved exactly (though numerically) as an algebraic system. This can be done for arbitrary $R$, without any use of the iterative approach. The price is, of course, that we deal with a nonlinear system of $3 n$ equations for $3 n$ variables, which is quite challenging even numerically. 


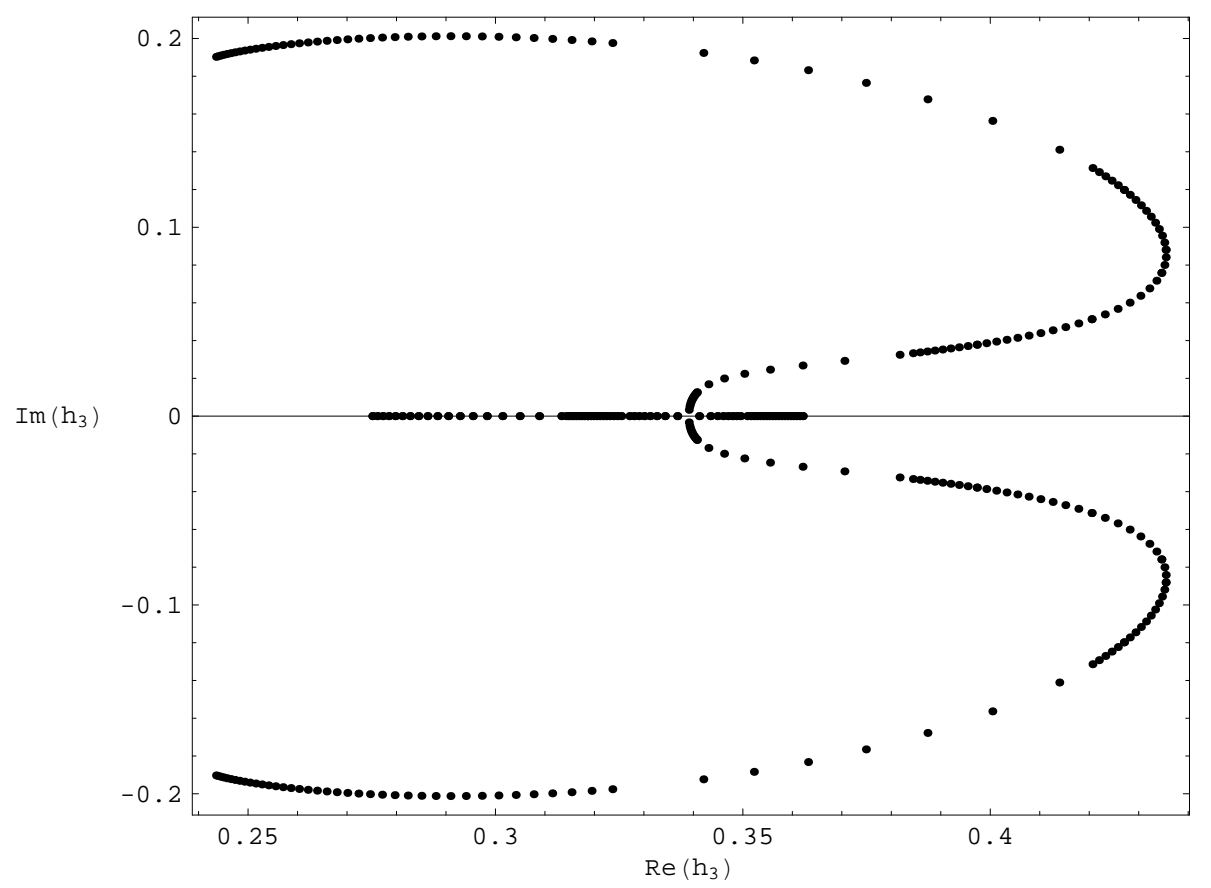

Figure 6: Two complex trajectories $h(k)$ (at $k=k_{3}=2$.) parametrized by $R$ moving through the critical point.

Restricting to identical sources reduces a problem to $n$ variables. Still there are $3^{n}$ solutions, and Mathematica has to generate all of them before we can choose the physically acceptable ones. To produce all 729 solutions for $n=6$ takes about 2.5 hours hence, in practice, the method is limited at present to $n \leq 5$. Still, even such a crude discretization reasonably reproduces the main features of, say, the momentum distribution. Therefore we go ahead with the simplified problem here and look for complex solutions above $R_{c}$ leaving the, obviously possible, refinements for the future.

Figure 6 shows the behaviour of two complex trajectories ${ }^{1}$ as $\mathrm{R}$ moves from the dispersive to the $\mathrm{BH}$ phase. We show only a discrete series of points with varying step $\Delta R$ (see below) to give also an idea of the speed (in $R$ ) along a trajectory. For $n=5$, the critical point is at $R_{c}=0.3193$. We begin by following the left trajectory (also reproduced by the recursion in this region) deep inside the dispersive phase (at $R=0.15$ ): it moves towards its critical value $x_{c}$ along the real axis, as expected. Checking the stability matrix at the fixed point we verified that, indeed, this is the only solution which is stable against the iterations (4.6). The second solution (which starts above $x_{c}$ in the figure) is also real, but unstable. We have chosen it to be the one that matches the recursive solution at $x_{c}$. The sampling points are separated by $\Delta R=0.01$ at the beginning of the recursive solution and then, as we approach $R_{c}$, the coarse graining was reduced to $\Delta R=0.001$. One readily sees that while approaching $x_{c}$ the variation w.r.t. $R$ increases in agreement with the findings of section 5 . For the unstable trajectory $\Delta R$ was set to 0.001 from the beginning since we

\footnotetext{
${ }^{1}$ By a trajectory we mean here a path traced by $h(k)$, at one value of $k$, while changing $R$.
} 
started tracing it much closer to $R_{c}$. Again, its velocity increases as we go towards the critical point. As soon as one goes above $R_{c}$ both trajectories acquire (complex conjugate) imaginary pats $y(R)$. Closer inspection shows that these imaginary parts grow like a square root, $\sqrt{x-x_{c}}$, as typical for a threshold behaviour. Since both trajectories are complex conjugate to each other, figure 6 implies that the real part of the left trajectory continues to increase while that of the right trajectory changes from decreasing to increasing as we go through $R_{c}$. Interestingly, the trajectories reveal a rich structure even when we go deeper inside the $\mathrm{BH}$ phase. We have followed them up to $R=0.65$ changing the R-resolution: $\Delta R=0.001 \rightarrow 0.01 \rightarrow 0.005 \rightarrow 0.01$ and finally to $\Delta R=0.003$ along the last segments. In these regions the trajectories appear to saturate. It remains to be seen if all these detailed features, like bending or saturation, are generic or are just artefacts of our small value of $n$. For example, we know that increasing $n$ would move $R_{c}$ by more than $30 \%$.

However, one thing is clear: for $R>R_{c}$ classical solutions of (4.6) (and consequently also the on shell effective action) develop imaginary parts. This is turn implies a new absorption of the elastic amplitude (on top of the one due to graviton production) calling for the opening of some new channel. The whole mechanism is somewhat reminiscent of the classic discussion of the "decay of the false vacuum" by Coleman and de Luccia [12 as a tunnelling process described through the contribution of complex saddle points to the functional integral. Use of similar ideas in this new context is presently under investigation [13].

Let us conclude by stressing that the non-linearities captured by equations (4.6) are essential for the above instabilities to occur. The gravitational attraction alone exists already in the lowest Born diagram, but it is not sufficient to produce the non-linearities of the metric that are essential for the buildup of CTS. Our results confirm that, instead, the class of diagrams selected in [2] appear to be sufficient for bringing out the emergence of such phenomena.

\section{Acknowledgments}

We wish to thank M. Ciafaloni for interesting discussions and for communicating to us some preliminary results from ref. [13] prior to publication. We also acknowledge the warm hospitality of the Galileo Galilei Institute in Arcetri (Florence, Italy) during the completion phase of this work.

Note added in proofs. Actually, eq. (2.12) can be proven by using the results of A. Gervois and H. Navelet, Jour. Math. Phys. 26 (1985) 633 (see their table 1). We are grateful to Dr. F. Piazza for pointing out to us the existence of this work.

\section{References}

[1] G. Veneziano and J. Wosiek, Exploring an S-matrix for gravitational collapse, arXiv:0804.3321.

[2] D. Amati, M. Ciafaloni and G. Veneziano, Towards an S-matrix description of gravitational collapse, JHEP 02 (2008) 049 arXiv:0712.1209. 
[3] D. Amati, M. Ciafaloni and G. Veneziano, Superstring collisions at Planckian energies, Phys. Lett. B 197 (1987) 81; Classical and quantum gravity effects from Planckian energy superstring collisions, Int. J. Mod. Phys. A 3 (1988) 1615.

[4] L.N. Lipatov, High-energy scattering in $Q C D$ and in quantum gravity and two-dimensional field theories, Nucl. Phys. B 365 (1991) 614;

R. Kirschner and L. Szymanowski, Effective action for high-energy scattering in gravity, Phys. Rev. D 52 (1995) 2333 hep-th/9412087.

[5] D. Amati, M. Ciafaloni and G. Veneziano, Effective action and all order gravitational eikonal at Planckian energies, Nucl. Phys. B 403 (1993) 707; Higher order gravitational deflection and soft bremsstrahlung in Planckian energy superstring collisions, Nucl. Phys. B 347 (1990) 550 .

[6] G. Marchesini and E. Onofri, High energy gravitational scattering: a numerical study, JHEP 06 (2008) 104 arXiv:0803.0250.

[7] D.M. Eardley and S.B. Giddings, Classical black hole production in high-energy collisions, Phys. Rev. D 66 (2002) 044011 gr-qc/0201034;

H. Yoshino and Y. Nambu, Black hole formation in the grazing collision of high- energy particles, Phys. Rev. D 67 (2003) 024009 [gr-qc/0209003];

S.B. Giddings and V.S. Rychkov, Black holes from colliding wavepackets, Phys. Rev. D 70 (2004) 104026 hep-th/0409131.

[8] E. Kohlprath and G. Veneziano, Black holes from high-energy beam-beam collisions, JHEP 06 (2002) 057 gr-qc/0203093.

[9] A.M. Abrahams and C.R. Evans, Critical behavior and scaling in vacuum axisymmetric gravitational collapse, Phys. Rev. Lett. 70 (1993) 2980; Universality in axisymmetric vacuum collapse, Phys. Rev. D 49 (1994) 3998.

[10] M.W. Choptuik, E.W. Hirschmann, S.L. Liebling and F. Pretorius, Critical collapse of the massless scalar field in axisymmetry, Phys. Rev. D 68 (2003) 044007 gr-qc/0305003.

[11] M.W. Choptuik, Universality and scaling in gravitational collapse of a massless scalar field, Phys. Rev. Lett. 70 (1993) 9;

for a review, see e.g. C. Gundlach, Critical phenomena in gravitational collapse, gr-qc/0210101.

[12] S.R. Coleman and F. De Luccia, Gravitational effects on and of vacuum decay, Phys. Rev. D 21 (1980) 3305.

[13] M. Ciafaloni and D. Colferai, S-matrix and quantum tunneling in gravitational collapse, arXiv:0807.2117. 